\title{
Long non-coding RNA HIT000218960 is associated with poor prognosis in patients with gastric cancer
}

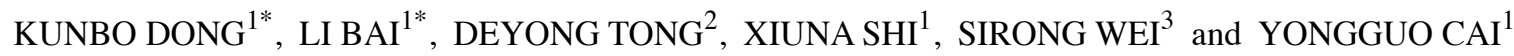 \\ Departments of ${ }^{1}$ Gastroenterology, ${ }^{2}$ Oncology and ${ }^{3}$ Intervention, The 970th Hospital of \\ the PLA joint Logistics Support Force, Yantai, Shandong 264001, P.R. China
}

Received March 31, 2020; Accepted February 15, 2021

DOI: $10.3892 /$ etm.2021.10126

\begin{abstract}
Long non-coding RNAs (lncRNAs) have been indicated to have important roles in the development of malignant tumors. In the present study, the expression of HIT000218960 in gastric cancer (GC) tissues was assessed and its clinical significance was analyzed. It was revealed that HIT000218960 was highly expressed in GC tissues and HIT000218960 levels in GC tissues from 103 cases were positively correlated with high mobility group AT-hook 2 (HMGA2) mRNA expression. Furthermore, HIT000218960 was significantly associated with tumor diameter, TNM stage, histological grade, the number of lymph nodes with metastasis and HMGA2 expression in tumor tissues of patients with GC. The results of the univariate and multivariate Cox regression analysis indicated that HIT000218960 expression was a factor affecting the prognosis of patients with GC. In addition, patients with GC with lower HIT000218960 or HMGA2 expression had more favorable 3-year survival, while HIT000218960 expression did not affect the 3-year overall survival of patients with GC with different levels of HMGA2 expression. In conclusion, HIT000218960 was highly expressed in patients with GC and was related to poor prognosis.
\end{abstract}

\section{Introduction}

Gastric cancer (GC) is one of the most common malignant tumor types of the digestive tract in the world. Japan, South Korea and China are regions with a high incidence of GC and $>600,000$ new cases are registered each year in China, accounting for $42 \%$ of newly registered cases in the world $(1,2)$.

Correspondence to: Dr Yongguo Cai, Department of Gastroenterology, The 970th Hospital of the PLA joint Logistics Support Force, 7 South Zhichu Road, Yantai, Shandong 264001, P.R. China

E-mail: caiyg7011@126.com

*Contributed equally

Key words: long non-coding RNA, HIT000218960, gastric cancer, prognosis
The mortality rate of patients with GC ranks among the top 3 of all malignancies and has not exhibited any downward trend (3). Although early detection and early treatment are the most effective measures to reduce the mortality of GC, the detection rate of early GC is only $10 \%$ and the lack of specific biomarkers for GC is a major obstacle (4).

Of the human genomic DNA (including introns and exons), $75 \%$ is transcribed into RNA, but only $2 \%$ of the genome encodes proteins and $98 \%$ of transcripts are non-coding RNAs $(5,6)$. A single-stranded RNA molecule with a length of about 20-24 nucleotides is called a non-coding single-stranded RNA molecule, and a non-coding RNA with a length of $>200$ nucleotides is called long non-coding RNA (lncRNA) $(5,6)$. IncRNA was originally considered to be 'junk' RNA, but in recent years, research has indicated that IncRNA has an important role in numerous biological processes such as dose compensation effects, epigenetic regulation and control of the cell cycle regulation and cell differentiation (7). In GC, increasing evidence has indicated that certain IncRNAs are involved in the proliferation, migration, invasion and evasion of apoptosis of GC cells, including lncRNA LINC00978 (8), lncRNA ZFAS1 (9) and lncRNA HAGLROS (10). lncRNA HIT000218960 is a newly discovered lncRNA and was reported to be highly expressed in papillary thyroid cancer (11) and GC (12). In addition, the expression levels of HIT000218960 were associated with tumor size, Tumor-Node-Metastasis (TNM) stage and lymph node metastasis in patients with GC (12). However, studies on lncRNA HIT000218960 in GC are rare. In the present study, HIT000218960 expression was detected in GC tissues from 103 cases and normal gastric mucosal tissues from 62 subjects and its prognostic value in patients with GC was assessed.

\section{Materials and methods}

Patients, tissues and grouping. Between January 2015 and June 2016, GC tissues from 103 cases and normal gastric mucosal tissues from 62 subjects were collected for analysis in the present study. GC tissues were collected from surgical removal of tumors and normal gastric mucosal tissues were collected from gastric mucosal biopsies of healthy individuals). The clinical data of the patients with GC are provided in Table I, and the age individuals providing normal gastric mucosal tissue was $55.2 \pm 13.8$ years (38-72 years), with 
25 males and 37 females. The inclusion criteria were as follows: i) Diagnosed with GC, ii) complete 3-year follow-up, iii) being informed of the content of the study and voluntarily joining the research. The exclusion criteria were as follows: i) Diagnosis with another malignant tumor type, severe cardio-cerebral vascular disease or organ dysfunction, ii) a lack of basic and 3 -year follow-up records, iii) death from other illnesses or an accident, iv) individuals who were pregnant, lactating or diagnosed with chronic viral (HIV, hepatitis B and/or C virus) or bacterial infections (M. tuberculosis), v) individuals who underwent chemotherapy, immunotherapy or targeted therapy prior to tissue acquisition. All patients were informed and signed an informed consent form. All experiments involving human tissues were approved and supervised by the Ethics Committee of the 970th Hospital of the PLA joint Logistics Support Force (Yantai, China). In addition, patients were divided into groups based on HIT000218960 expression and HMAG2 mRNA expression: The low HMGA2 expression group $(n=53)$ consisted of patients with HMGA2 expression levels $\leq$ median of the HMGA20 expression levels (2.505) and the high HMGA2 expression group $(\mathrm{n}=50)$ consisted of patients with HIT000218960 expression levels > median of the HMGA2 expression.

Reverse transcription-quantitative (RT-q)PCR analysis. Total RNA was extracted $\mathrm{f} \mu \mathrm{rom}$ the tissues using RNAiso plus (cat. no. 9108; Takara Bio, Inc.). After preparing the complementary DNA using a PrimeScript RT reagent kit with gDNA eraser (cat. no. RR047A; Takara Bio, Inc.), $20 \mu \mathrm{l}$ of a qPCR mixture were prepared using GoTaq qPCR Master Mix (cat. no. A6001; Promega, Corp.) according to the manufacturers' protocols for both RT and PCR using Touch system (CFX384; Bio-Rad Laboratories, Inc.) and the thermocycling conditions were showed as follows: $95^{\circ} \mathrm{C}$ of $2 \mathrm{~min},\left(95^{\circ} \mathrm{C}\right.$ of $5 \mathrm{sec}$ and $65 \mathrm{C}$ of $15 \mathrm{sec}$ ) for 40 cycles. The primer sequences were asfollows: $\beta$-actinforward, 5'-AGCCCATCCTTCGAGTA CAAA-3' and reverse, 5'-TCTTGGTGCGATAACTGGTGG-3'; HIT000218960 forward, 5'-CCACCTACCCATCTGAC TTTG-3' and reverse, 5'-CCACTATTTCCCACTGCCTT-3'; high mobility group AT-hook 2 (HMGA2) forward, 5'-ACCCA GGGGAAGACCCAAA-3' and reverse, 5'-CCTCTTGGCC GTTTTTCTCCA-3'. The relative expression of HIT000218960 and HMGA2 mRNA were calculated with the $2^{-\Delta \Delta C q}$ method (13) and $\beta$-actin was used as a housekeeping gene.

Immunohistochemistry. Paraffin sections that were $4-\mu \mathrm{m}$ thick of preserved GC tumor tissue and normal gastric mucosal tissues were subjected to immunohistochemistry for the detection of the expression of HMGA2 protein. HMGA2 staining was performed in accordance with the instructions of the VECTASTAIN ${ }^{\circledR}$ Elite $^{\circledR}$ ABC Kit (Vector Laboratories, Inc.). Anti-HMGA2 antibody (1:500 dilution; cat. no. ab207301; Abcam) was selected as the primary antibody and goat Anti-Rabbit IgG H\&L (HRP) (1:2,000 dilution; cat. no. ab6702; Abcam) was selected as the secondary antibody. Samples were incubated with primary antibody overnight at $4^{\circ} \mathrm{C}$ and with secondary antibody for $2 \mathrm{~h}$ at $37^{\circ} \mathrm{C}$, and then the slices were soaked in hematoxylin and stained for $30 \mathrm{sec}$ at room temperature. Finally, slices were analyzed using a Leica TCS SP5 microscope (Leica Microsystems) with the LAS AF Lite 4.0 image browser software (Leica Microsystems) and the HMGA2-positive cells were recorded.

Statistical analysis. GraphPad Prism 5 software was used for data analysis. Differences between two groups were analyzed using the Student's t-test or the $\chi^{2}$ test, and differences between multiple groups were assessed using one-way ANOVA with Tukey's test as the post-hoc test. The log-rank (Mantel-Cox) test was used to compare the survival of patients with GC. The Pearson correlation coefficient was used to measure the correlation between two variables in the cohort. The Cox regression model was employed for analyzing factors that affect the survival of patients with GC. $\mathrm{P}<0.05$ was considered to indicate statistical significance.

\section{Results}

HIT000218960 is highly expressed in GC tissues. Normal tissues from a total of 62 subjects and GC tissues from 103 cases were collected and used to detect HIT000218960 expression by RT-qPCR. As presented in Fig. 1A, the expression of HIT000218960 in GC tissues was significantly higher than that in normal tissues. Next, HIT000218960 expression was compared among different subgroups of patients with GC based on clinical features and significant differences were obtained among patients with different histological grades (Fig. 1B), TNM stages (Fig. 1C) and numbers of lymph nodes with metastasis (Fig. 1D). The expression of HIT000218960 was lowest in 28 cases of histological grade I and in 17 cases of histological grade III, it was the highest (Fig. 1B). Furthermore, in 41 cases of TNM stage-II, 48 cases of stage-III and 14 cases of stage-IV GC, HIT000218960 expression increased gradually with the TNM stage (Fig. 1C). According to the number of lymph nodes with metastases, the 103 cases of patients with GC were divided into 3 groups with $0(n=18), 1-6(n=48)$ and $\geq 7$ ( $n=37$ ) lymph nodes with metastases. As presented in Fig. 1D, HIT000218960 expression increased gradually with the number of lymph nodes with metastases in patients with GC.

HIT000218960 expression is related to HMGA2. HMGA2 is an oncogene for GC and has been indicated to be elevated in GC tissues $(14,15)$. In the present study, the expression of HMGA2 was first detected in normal tissues and GC tissues and the correlation between HIT000218960 expression and HMGA2 expression was then analyzed to assess the significance of HIT000218960 expression in GC tissues. The data of the RT-qPCR analysis suggested that the expression of HMGA2 mRNA in GC tissues was significantly higher than that in normal tissues (Fig. 2A). Similarly, the results of the immunohistochemistry analysis indicated that the expression of HMGA2 protein in GC tissues was also significantly higher than that in normal tissues (Fig. 2B). Of note, the correlation between HIT000218960 expression and HMGA2 mRNA expression in GC tissues was analyzed and it was revealed that there was a positive correlation between HIT000218960 expression and HMGA2 mRNA expression in GC tissues (Fig. 2C).

Clinical significance of HIT000218960 expression. To further evaluate the clinical significance of HIT000218960 expression 
Table I. HIT000218960 expression and clinical data of patients with gastric cancer.

\begin{tabular}{|c|c|c|c|c|c|}
\hline \multirow[b]{2}{*}{ Item } & \multicolumn{2}{|c|}{ HIT000218960 expression } & \multirow[b]{2}{*}{ High $(n=47)$} & \multirow[b]{2}{*}{$\chi^{2}$} & \multirow[b]{2}{*}{ P-value } \\
\hline & Cases (n) & Low $(n=56)$ & & & \\
\hline Sex & & & & 0.920 & 0.337 \\
\hline Female & 65 & 33 & 32 & & \\
\hline Male & 38 & 23 & 15 & & \\
\hline Age (years) & & & & 0.690 & 0.406 \\
\hline$\geq 60$ & 59 & 30 & 29 & & \\
\hline$<60$ & 44 & 26 & 18 & & \\
\hline Tumor diameter $(\mathrm{cm})$ & & & & 6.756 & 0.009 \\
\hline$\geq 3$ & 65 & 29 & 36 & & \\
\hline$<3$ & 38 & 27 & 11 & & \\
\hline TNM stage & & & & 10.914 & 0.004 \\
\hline II & 41 & 29 & 12 & & \\
\hline III & 48 & 24 & 24 & & \\
\hline IV & 14 & 3 & 11 & & \\
\hline Histological grade & & & & 6.336 & 0.042 \\
\hline I & 28 & 19 & 9 & & \\
\hline II & 58 & 32 & 26 & & \\
\hline III & 17 & 5 & 12 & & \\
\hline Lymph node metastasis (n) & & & & 13.195 & 0.004 \\
\hline 0 & 18 & 15 & 3 & & \\
\hline $1-2$ & 20 & 13 & 7 & & \\
\hline $3-6$ & 28 & 15 & 13 & & \\
\hline$\geq 7$ & 37 & 13 & 25 & & \\
\hline HMGA2 expression & & & & 27.234 & $<0.001$ \\
\hline Low & 53 & 42 & 11 & & \\
\hline High & 50 & 14 & 36 & & \\
\hline
\end{tabular}

HMGA2, high mobility group AT-hook 2.

in patients with GC, the 103 cases of GC were divided into 2 groups based on HIT000218960 expression, namely the low HIT000218960 expression group $(\mathrm{n}=56$; HIT000218960 expression levels $\leq$ median of the HIT000218960 expression levels in 103 the GC patients) and the high HIT000218960 expression group ( $n=47$; HIT000218960 expression levels $>$ median of the HIT000218960 expression levels in the 103 GC patients). At the same time, GC patients also were divided into 2 groups based on HMGA2 mRNA and HIT000218960 expression. As presented in Table I, the expression of HIT000218960 in the 103 cases of GC was not significantly associated with sex $(\mathrm{P}=0.337)$ or age $(\mathrm{P}=0.406)$, while it was significantly related to tumor diameter $(\mathrm{P}=0.009)$, TNM stage $(\mathrm{P}=0.004)$, histological grade $(\mathrm{P}=0.042)$, the number of lymph nodes with metastasis $(\mathrm{P}=0.004)$ and HMGA2 expression $(\mathrm{P}<0.001)$.

HIT000218960 is associated with the prognosis of patients with $G C$. Survival prognosis is the best indicator for tumor patients to obtain treatment benefits and thus, the prognostic significance of HIT000218960 expression in GC patients was assessed by analyzing factors affecting prognosis. Following univariate logistic regression analysis, the results of the multivariate Cox regression analysis indicated that TNM stage (hazard ratio $=2.658,95 \% \mathrm{CI}=0.891-3.254)$, histological grade $(\mathrm{OR}=1.026,95 \% \mathrm{CI}=0.658-1.627)$, lymph node metastasis $(\mathrm{OR}=2.232,95 \% \mathrm{CI}=1.241-3.654)$, HIT000218960 expression $(\mathrm{OR}=1.567,95 \% \mathrm{CI}=1.118-3.567)$ and HMGA2 expression $(\mathrm{OR}=2.892,95 \% \mathrm{CI}=1.387-4.125)$ were factors affecting the prognosis of patients with GC (Table II). Next, the 3-year survival of patients with GC with different HIT000218960 and HMGA2 expression was compared. As presented in Fig. 3, the 3-year survival of patients with GC with low HIT000218960 expression was significantly higher than that in patients with high HIT000218960 expression (Fig. 3A). Among the 56 cases of GC with low HIT000218960 expression, the 3 -year survival of patients with low HMGA2 expression was significantly higher than that of patients with high HMGA2 expression (Fig. 3B), and similar results were also obtained for the 47 cases of GC with high HIT000218960 expression (Fig. 3C). Similarly, the 3-year survival of patients with GC and low HMGA2 expression was significantly higher than that of patients with high HMGA2 expression (Fig. 3D). However, 

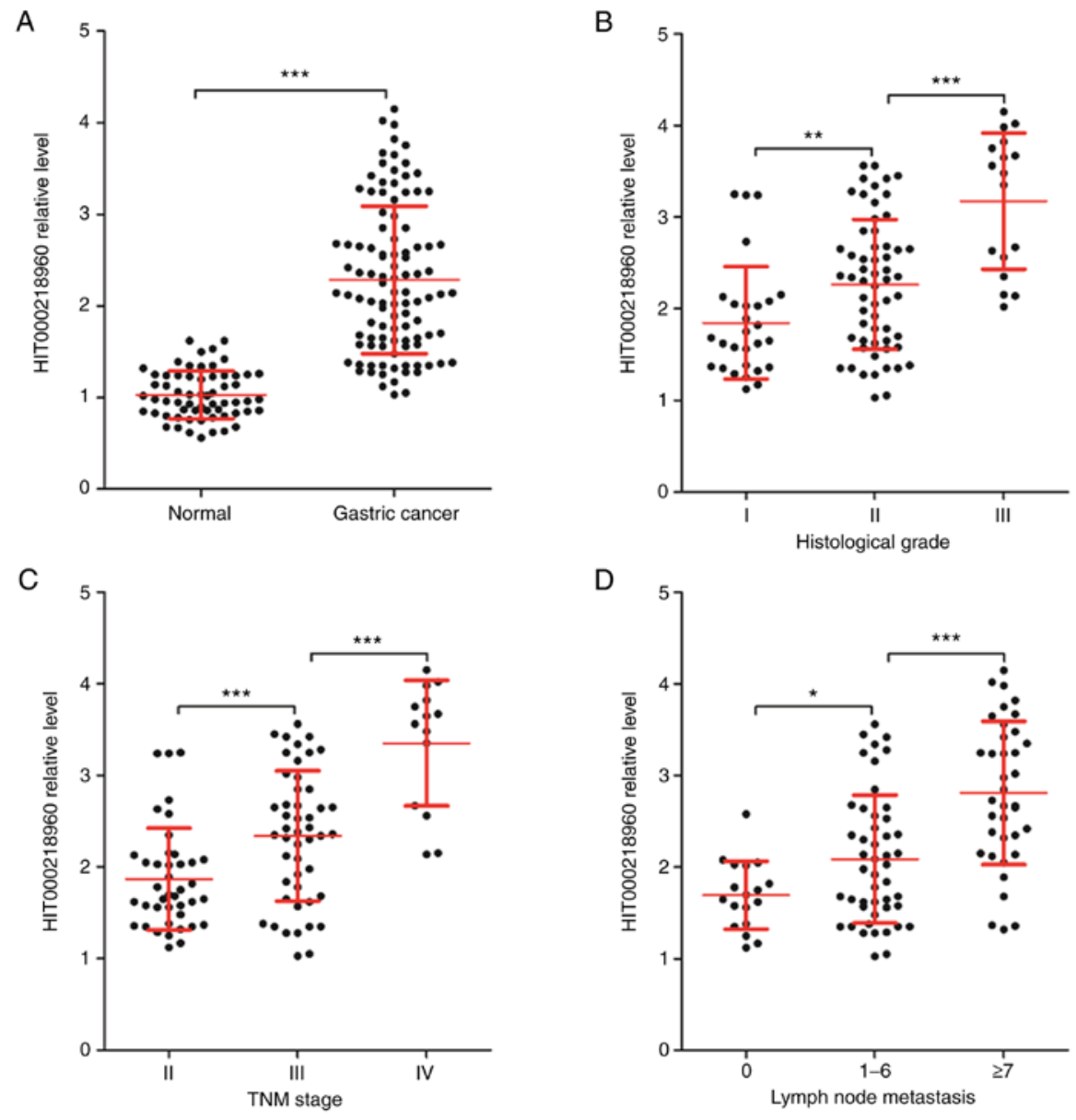

Figure 1. HIT000218960 expression in GC tissues. (A) Reverse transcription-quantitative PCR analysis of HIT000218960 levels in normal gastric tissues from 62 subjects and GC tissues 103 from cases. Statistical comparison of HIT000218960 levels among cases of GC with different (B) histological grades, (C) TNM stages and (D) number of lymph nodes with metastasis. Values are expressed as the mean \pm standard deviation. ${ }^{*} \mathrm{P}<0.05,{ }^{* * *} \mathrm{P}<0.01$ and ${ }^{* * * *} \mathrm{P}<0.001$. GC, gastric cancer.
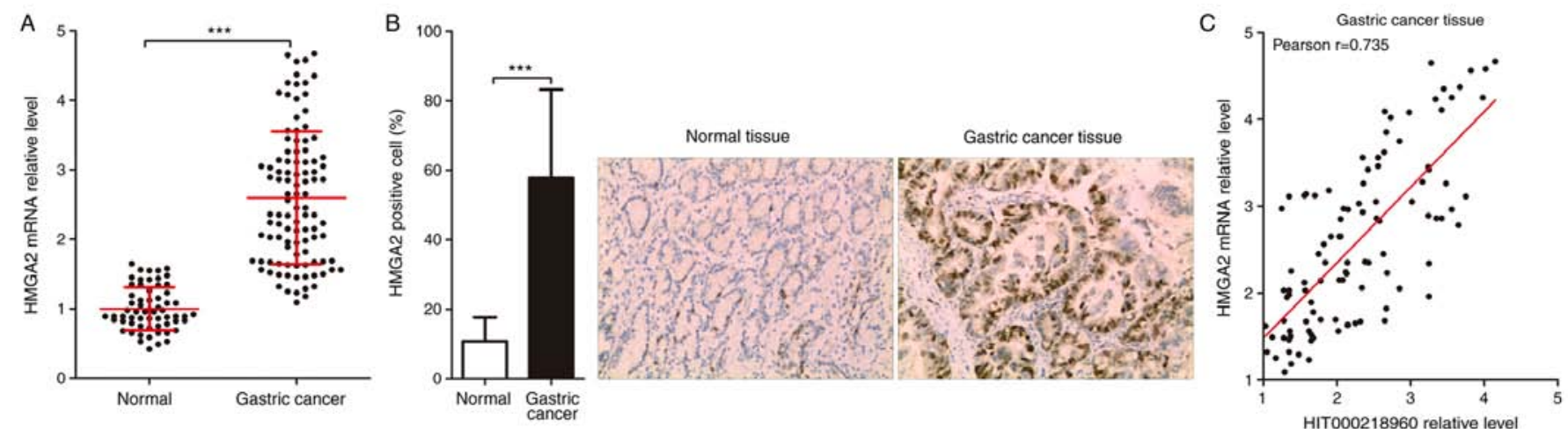

Figure 2. Correlation between HIT000218960 and HMGA2 expression in patients with GC. (A) Reverse transcription-quantitative PCR analysis of the indicated HMGA2 mRNA levels in normal tissues from 62 subjects and GC tissues from 103 cases. (B) Immunohistochemistry was used to determine HMGA2 protein expression in normal tissues $(n=62)$ and GC tissues $(n=103)$. The immunohistochemical score is provided on the left and representative immunohistochemical staining images (magnification, x100) are provided on the right. (C) Correlation analysis of HIT000218960 and HMGA2 expression in GC patients. The r-value was calculated by the Pearson method. Values are expressed as the mean \pm standard deviation. ${ }^{* * *} \mathrm{P}<0.001$. HMGA2, high mobility group AT-hook 2; GC, gastric cancer.

there was no significant difference in survival between GC patients with low and high HIT000218960 expression, whether in the group of 53 cases with GC low HMGA2 expression (Fig. 3E) or among the 50 cases of GC with high HMGA2 expression (Fig. 3F).

\section{Discussion}

According to data released by the China National Cancer Center (3), the morbidity and mortality of GC rank second among all malignancies in males and 679,000 new cases and 
Table II. Univariate and multivariate Cox regression analysis for the prognosis of patients with gastric cancer.

\begin{tabular}{|c|c|c|c|c|c|c|}
\hline \multirow[b]{2}{*}{ Clinical parameter } & \multicolumn{3}{|c|}{ Univariate analysis } & \multicolumn{3}{|c|}{ Multivariate analysis } \\
\hline & OR & $95 \% \mathrm{CI}$ & P-value & OR & $95 \% \mathrm{CI}$ & P-value \\
\hline Sex (males vs females) & 1.032 & $0.569-2.031$ & 0.632 & - & - & - \\
\hline Age $(\geq 60$ vs $<60)$ & 0.628 & $0.235-1.351$ & 0.289 & - & - & - \\
\hline Tumor diameter, $\geq 3$ vs $<3$ & 2.325 & $1.256-3.541$ & 0.068 & - & - & - \\
\hline TNM stage, II vs III+IV & 3.021 & $1.568-4.025$ & $<0.001$ & 2.658 & $0.891-3.254$ & $<0.001$ \\
\hline Histological grade, I vs II+III & 2.541 & $1.335-2.987$ & 0.021 & 1.026 & $0.658-1.627$ & 0.038 \\
\hline Lymph node metastasis, yes vs no & 1.524 & $0.985-3.127$ & 0.005 & 2.232 & $1.241-3.654$ & 0.002 \\
\hline HIT000218960 expression, low vs high & 1.023 & $0.569-1.352$ & 0.038 & 1.567 & $1.118-3.567$ & 0.042 \\
\hline HMGA2 expression, low vs high & 1.953 & $1.055-3.246$ & $<0.001$ & 2.892 & $1.387-4.125$ & 0.003 \\
\hline
\end{tabular}

SPSS statistical analysis software was used for multivariate Cox regression analysis. When P $>0.05$, the OR and $95 \%$ CI were not displayed by the software. HMGA2, high mobility group AT-hook 2; OR, odds ratio.
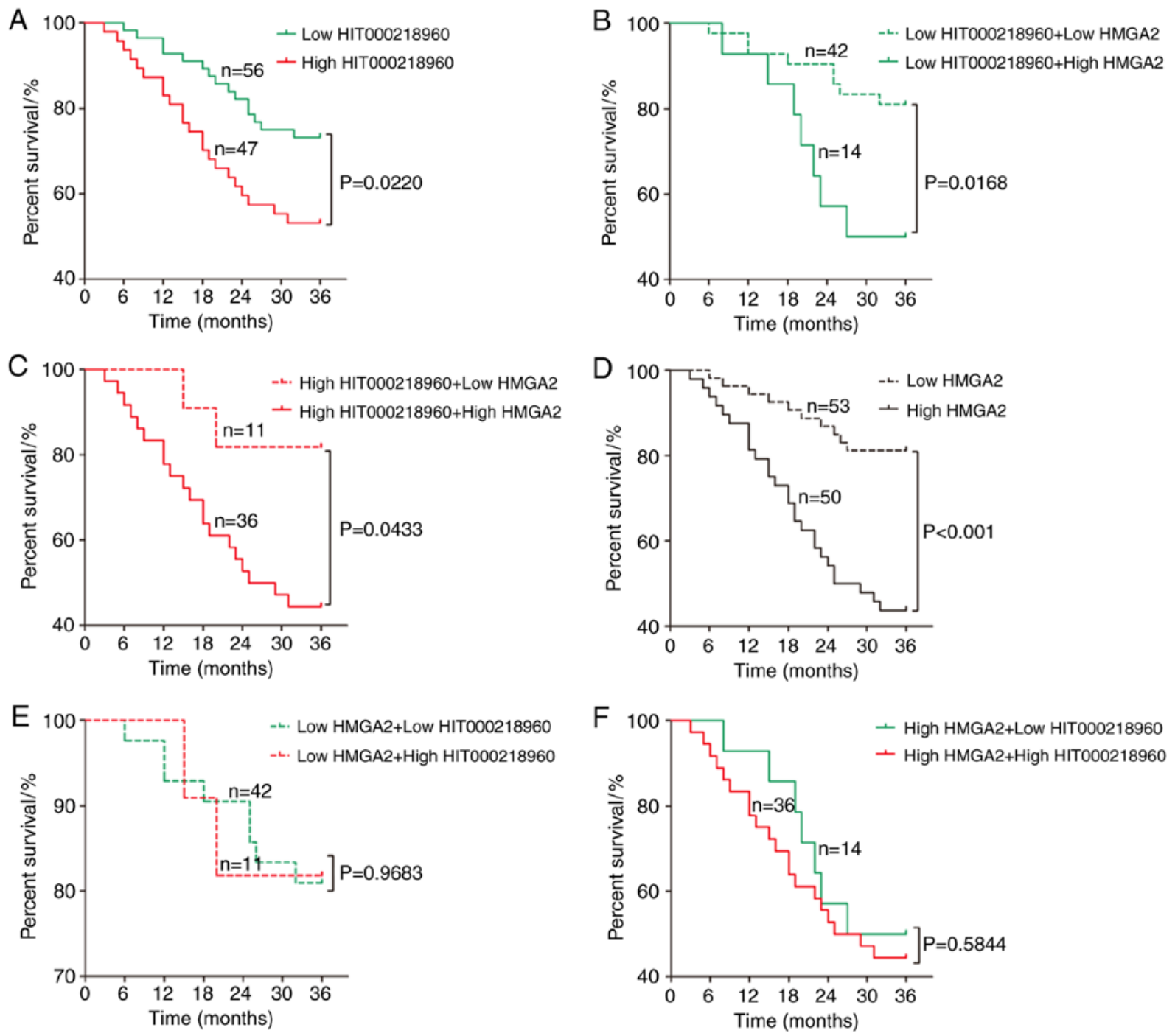

Figure 3. HIT000218960 predicted that the poor prognosis of patients with GC was related to HMGA2 expression. (A) Survival (3-year) of patients with GC were compared based on different HIT000218960 expression. (B) Survival (3-year) of patients with GC with low HIT000218960 expression were compared based on different HMGA2 expression. (C) Survival (3-year) of patients with GC with high HIT000218960 expression were compared based on different HMGA2 expression levels. (D) Survival (3-year) of patients with GC were compared based on different HMGA2 expression levels. (E) Survival (3-year) of patients with GC in low HMGA2 expression were compared based on different HIT000218960 expression levels. (F) Survival (3-year) of patients with GC with high HMGA2 expression were compared based on different HIT000218960 expression levels. The P-value was calculated with the log-rank (Mantel-Cox) test. HMGA2, high mobility group AT-hook 2; GC, gastric cancer. 
60,000 deaths are reported each year. Of note, most patients with GC are already at an advanced stage at the time-point of diagnosis, and thus, the 5-year survival for patients with GC is low, e.g. the 5-year overall survival rate of patients with GC treated only by surgery is $\sim 20 \%$ and $30-50 \%$ in patients treated by surgery and adjuvant therapy $(16,17)$. Therefore, it may be worthwhile to identify biomarkers that may be used to diagnose and evaluate the prognosis of patients with GC. In the present study, it was not only determined that HIT000218960 was highly expressed in GC tissue, but also that HIT000218960 expression increased with the degree of GC differentiation, clinical stage and number of lymph nodes with metastases. A recent similar study suggested that HIT000218960 was highly expressed in GC as compared to normal tissues adjacent to GC tissues (12). Furthermore, a previous study suggested that HIT000218960 was highly expressed in papillary thyroid cancer tissues and promoted the proliferation and metastasis of papillary thyroid cancer cells in vitro (11). Of note, these two studies all indicated that the function of HIT000218960 in cancer cells was related to HMGA2.

HMGA2 has been recognized as a novel oncogene and HMGA2 protein is a non-histone chromatin-related protein that may change its structure by binding to chromatin or directly interact with related proteins to regulate the transcription of other genes to then induce tumor formation $(18,19)$. HMGA2 was reported to be involved in numerous aspects of the biological characteristics of GC, such as proliferation (14), epithelial-mesenchymal transition $(15,20)$, migration and invasion $(21,22)$, chemical resistance $(23)$, tumor angiogenesis $(21,24)$ and tumor stem cell characteristics $(25)$. In the present study, HMGA2 mRNA expression was detected using RT-qPCR and HMGA2 protein expression using immunohistochemistry, and it was determined that HMGA2 was highly expressed in GC tissues, which was consistent with previous studies $(15,20)$. Furthermore, the expression of HIT000218960 was positively correlated with the expression of HMGA2 mRNA in GC tissues. This suggested that HIT000218960 may be an oncogene in GC.

To further explore the significance of HIT000218960 expression in patients with GC, the association between HIT000218960 expression and clinical features of GC was explored. As expected, HIT000218960 was significantly associated with clinical stage, tumor size, lymph node metastasis and HMGA2 expression in GC, and HIT000218960 was a factor affecting the prognosis of patients with GC. Furthermore, the effect of HMGA2 expression on the 3-year survival of patients with GC in HIT000218960 low or high expression was compared and significant differences were obtained, that was patients with GC with lower HIT000218960 or HMGA2 expression had more favorable 3 -year survival. However, insignificant differences were obtained when comparing the effect of HIT000218960 expression on the 3-year survival of patients with GC with HMGA2 low or high expression individually.

As an IncRNA, HIT000218960 does not directly regulate biological processes of cells and it may only have a biological function by directly interacting with the mRNA encoding a protein or by indirectly regulating the expression of a protein by binding to the microRNA $(26,27)$. Previous studies indicated that HIT000218960 promoted HMGA2 protein expression in GC and papillary thyroid cancer $(11,12)$. Therefore,
HIT000218960 may affect the biological characteristics of GC cells through HMGA2 and then affect the progression and prognosis of patients with GC. Overall, the present study suggested that HIT000218960 was highly expressed and was related to poor prognosis in patients with GC.

\section{Acknowledgements}

Not applicable.

\section{Funding}

No funding was received.

\section{Availability of data and materials}

The datasets used and/or analyzed during the current study are available from the corresponding author on reasonable request.

\section{Authors' contributions}

YC, LB and KD conceived and designed the study. LB and KD collected data, drafted the manuscript and critically revised the manuscript for the important intellectual content. DT, XS and SW analyzed and interpreted the experimental data. YC and LB confirm the authenticity of all the raw data. All authors read and approved the final version of the study.

\section{Ethics approval and consent to participate}

The present study was performed with the approval of the Ethics Committee of The 970th Hospital of the PLA joint Logistics Support Force (Yantai, China).

\section{Patient consent for publication}

Not applicable.

\section{Competing interests}

The authors declare that they have no competing interests.

\section{References}

1. Ferlay J, Soerjomataram I, Dikshit R, Eser S, Mathers C, Rebelo M, Parkin DM, Forman D and Bray F: Cancer incidence and mortality worldwide: Sources, methods and major patterns in GLOBOCAN 2012. Int J Cancer 136: E359-E386, 2015.

2. Bertuccio P, Chatenoud L, Levi F, Praud D, Ferlay J, Negri E, Malvezzi M and La Vecchia C: Recent patterns in gastric cancer: A global overview. Int J Cancer 125: 666-673, 2009.

3. Chen M, Wang W, Ma J, Ye P and Wang K: High glucose induces mitochondrial dysfunction and apoptosis in human retinal pigment epithelium cells via promoting SOCS1 and Fas/FasL signaling. Cytokine 78: 94-102, 2016.

4. Shiraishi N, Yasuda K and Kitano S: Laparoscopic gastrectomy with lymph node dissection for gastric cancer. Gastric Cancer 9: 167-176, 2006.

5. Zhao Y, Li H, Fang S, Kang Y, Wu W, Hao Y, Li Z, Bu D, Sun N, Zhang MQ, et al: NONCODE 2016: An informative and valuable data source of long non-coding RNAs. Nucleic Acids Res 44D1: D203-D208, 2016.

6. Ulitsky I: Evolution to the rescue: Using comparative genomics to understand long non-coding RNAs. Nat Rev Genet 17: 601-614, 2016. 
7. Mercer TR, Dinger ME and Mattick JS: Long non-coding RNAs: Insights into functions. Nat Rev Genet 10: 155-159, 2009.

8. Fu M, Huang Z, Zang X, Pan L, Liang W, Chen J, Qian H, $\mathrm{Xu}$ W, Jiang P and Zhang X: Long noncoding RNA LINC00978 promotes cancer growth and acts as a diagnostic biomarker in gastric cancer. Cell Prolif 51: e12425, 2018.

9. Pan L, Liang W, Fu M, Huang ZH, Li X, Zhang W, Zhang P, Qian H, Jiang PC, Xu WR, et al: Exosomes-mediated transfer of long noncoding RNA ZFAS1 promotes gastric cancer progression. J Cancer Res Clin Oncol 143: 991-1004, 2017.

10. Chen J-F, Wu P, Xia R, Yang J, Huo XY, Gu DY, Tang CJ, De $\mathrm{W}$ and Yang F: STAT3-induced lncRNA HAGLROS overexpression contributes to the malignant progression of gastric cancer cells via mTOR signal-mediated inhibition of autophagy. Mol Cancer 17: 6, 2018.

11. Li T, Yang XD, Ye CX, Shen ZL, Yang Y, Wang B, Guo P, Gao ZD, Ye YJ, Jiang KW, et al: Long noncoding RNA HIT000218960 promotes papillary thyroid cancer oncogenesis and tumor progression by upregulating the expression of high mobility group AT-hook 2 (HMGA2) gene. Cell Cycle 16 : 224-231, 2017.

12. Sun L, Yu J, Wang P, Shen M and Ruan S: HIT000218960 promotes gastric cancer cell proliferation and migration through upregulation of HMGA2 expression. Oncol Lett 17: 4957-4963, 2019.

13. Livak K J and Schmittgen TD: Analysis of relative gene expression data using real-time quantitative PCR and the $2^{-\Delta \Delta C T}$ method. Methods 25: 402-408, 2001.

14. Li W, Li J, Mu H, Guo M and Deng H: miR-503 suppresses cell proliferation and invasion of gastric cancer by targeting HMGA2 and inactivating WNT signaling pathway. Cancer Cell Int 19: $164,2019$.

15. Dong J, Wang R, Ren G, Li X, Wang J, Sun Y, Liang J, Nie Y, Wu K, Feng B, et al: HMGA2-FOXL2 Axis Regulates Metastases and Epithelial-to-Mesenchymal Transition of Chemoresistant Gastric Cancer. Clin Cancer Res 23: 3461-3473, 2017.

16. Jiang L, Yang KH, Guan QL, Chen Y, Zhao P and Tian JH: Survival benefit of neoadjuvant chemotherapy for resectable cancer of the gastric and gastroesophageal junction: A meta-analysis. J Clin Gastroenterol 49: 387-394, 2015.

17. Katai H, Ishikawa T, Akazawa K, Isobe Y, Miyashiro I, Oda I, Tsujitani S, Ono H, Tanabe S, Fukagawa T, et al; Registration Committee of the Japanese Gastric Cancer Association: Five-year survival analysis of surgically resected gastric cancer cases in Japan: A retrospective analysis of more than 100,000 patients from the nationwide registry of the Japanese Gastric Cancer Association (2001-2007). Gastric Cancer 21: 144-154, 2018.
18. Zhang S, Mo Q and Wang X: Oncological role of HMGA2 (Review). Int J Oncol 55: 775-788, 2019.

19. Ahmed SM, Ramani PD, Wong SQR, Zhao X, Ivanyi-Nagy R, Leong TC, Chua C, Li Z, Hentze H, Tan IB, et al: The chromatin structuring protein HMGA2 influences human subtelomere stability and cancer chemosensitivity. PLoS One 14: e0215696, 2019.

20. Zhao X-P, Zhang H, Jiao J-Y, Tang D-X, Wu YL and Pan C-B: Overexpression of HMGA2 promotes tongue cancer metastasis through EMT pathway. J Transl Med 14: 26, 2016.

21. Sun J, Sun B, Zhu D, Zhao X, Zhang Y, Dong X, Che N, Li J, Liu F, Zhao N, et al: HMGA2 regulates CD44 expression to promote gastric cancer cell motility and sphere formation. Am J Cancer Res 7: 260-274, 2017.

22. Wang H, Jiang Z, Chen $\mathrm{H}, \mathrm{Wu} \mathrm{X}$, Xiang $\mathrm{J}$ and Peng J: MicroRNA-495 inhibits gastric cancer cell migration and invasion possibly via targeting high mobility group AT-Hook 2 (HMGA2). Med Sci Monit 23: 640-648, 2017.

23. Yang X, Zhao Q, Yin H, Lei X and Gan R: miR-33b-5p sensitizes gastric cancer cells to chemotherapy drugs via inhibiting HMGA2 expression. J Drug Target 25: 653-660, 2017.

24. Lee MY, da Silva B, Ramirez DC and Maki RG: Novel HMGA2-YAP1 fusion gene in aggressive angiomyxoma. BMJ Case Rep 12: e227475, 2019.

25. Li W, Wang Z, Zha L, Kong D, Liao G and Li H: HMGA2 regulates epithelial-mesenchymal transition and the acquisition of tumor stem cell properties through TWIST1 in gastric cancer. Oncol Rep 37: 185-192, 2017.

26. Engreitz JM, Haines JE, Perez EM, Munson G, Chen J, Kane M, McDonel PE, Guttman M and Lander ES: Local regulation of gene expression by lncRNA promoters, transcription and splicing. Nature 539: 452-455, 2016.

27. Ferrè F, Colantoni A and Helmer-Citterich M: Revealing protein-IncRNA interaction. Brief Bioinform 17: 106-116, 2016.

This work is licensed under a Creative Commons Attribution-NonCommercial-NoDerivatives 4.0 International (CC BY-NC-ND 4.0) License. 